\title{
CCD sequences in the Shapley supercluster of galaxies. II. The ESO data $^{\star}$
}

\author{
E. Slezak ${ }^{1}$, L. Infante ${ }^{2}$ and H. Quintana $a^{2, \star \star}$ \\ 1 Observatoire de la Côte d'Azur, BP. 229, 06304 Nice Cedex 4, France \\ 2 Astronomy and Astrophysics Dept., P. Universidad Católica de Chile, Casilla 104, Santiago 22, Chile
}

Received March 21; accepted March 25, 1996

\begin{abstract}
We present $V$ and $R$ CCD photometric sequences of stars and galaxies for calibrating ESO/SERC (R) survey plates in the region of the Shapley supercluster of galaxies. ${ }^{\star \star \star}$
\end{abstract}

Key words: techniques: photometric — galaxies: photometry — surveys — galaxies: cluster of: Shapley supercluster

\section{Introduction}

One of the largest overdense region of the local Universe seems to be the very rich concentration of galaxies and of clusters of galaxies located in the northern Galactic hemisphere within $10^{\circ}$ of A3558 at $c z \sim 14000 \mathrm{~km} \mathrm{~s}^{-1}$. Since the late seventies our group is investigating the structure of this so-called Shapley supercluster by means of photometric and spectroscopic observations, these efforts resulting in redshifts, velocity dipersions and masses for a dozen of its clusters (see Quintana et al. 1995, and references therein). Renewed interest has been devoted to this region more recently, leading to a photometric catalogue of galaxies quite complete down to $b_{j} \simeq 17$ (Raychaudhury 1989) and to velocity measurements in the most inner part of the concentration (Bardelli et al. 1994).

We decided in 1992 to extend our study of the Shapley area by i) continuing our spectroscopic survey of the brightest galaxies $\left(r_{\mathrm{F}}<17.5\right)$ in a field of $5^{\circ}$ in radius around A3558 as well as of neighbouring clusters, and ii) by obtaining a homogeneous catalogue of galaxies of the whole $10^{\circ}$ radius region in a bandpass complementary to Raychaudhury's (1989) work where fainter objects will be detected to get a more complete view of the supercluster. A better understanding of the subclustering and dynamical properties of gravitationaly bound structures is in fact provided by taking into account their faint galaxy population. Crucial information with respect to competing

Send offprint requests to: E. Slezak

${ }^{\star}$ Based on observations collected at the European Southern Observatory, La Silla, Chile

${ }^{\star \star}$ Presidential Chair in Science

${ }^{\star \star \star}$ Tables 2 to 38 are available at the CDS via anonymous ftp 130.79.128.5 models for the formation of galaxies are also provided by various indicators when measured at scales large enough from a statistical sample. The amplitude of the cosmological signal at these scales is however very small and no reliable estimates can be obtained if some systematic biases occur in the survey. So, deep catalogues and consistent photometry over large scales are required.

Even if large digital sky surveys and their spectroscopic follow-ups are planned for the next future, twodimensional counts of galaxies obtained from Schmidt plates analysis are still of interest for investigating the large-scale properties of their distribution. But such photographic emulsions suffer from a non linear and spatialvarying response, and it is then quite difficult to get an accurate photometric calibration unless a monitoring of these variations is available from many CCD frames. We decided to secure such an extensive calibration for our photometric catalogue of the Shapley Supercluster derived from scans of 16 fields of the $\mathrm{ESO} / \operatorname{SERC}(\mathrm{R})$ survey performed by the MAMA facility in Paris. The small number of plates involved in this project enabled us to plan to obtain CCD sequences for 9-13 regularly distributed spots per plate, thereby mapping in a quite accurate way the pattern of each emulsion response while keeping the requested observational time within reach. Furthermore, the overlap between the $\mathrm{R}$ plates being small and too close to their edges for a meaningful scaling of each individual count, a dedicated set of wider digital data within the overlapping areas was also planned in order to get a homogeneous catalogue over the overall Shapley region. As stated by Cunow (1993) in a similar context, we are only interested in good photometric zero points for a large number of fields rather than trying to achieve high photometric quality for each object. Besides, we have to deal with stars 
only since the pixel data from the MAMA scans have been linearized according to the internal calibration procedure described by Bunclark \& Irwin (1983). After completion, the whole set of calibration magnitudes will be connected to the photographic $r_{\mathrm{F}}$ bandpass of the $\mathrm{ESO} / \mathrm{SERC}(\mathrm{R})$ plates and a $2 D$ spline fitting will be performed over the local photometric zero-points to compute its exact value at any other location.

The purpose of this paper is to present the CCD sequences obtained with the $2.2 \mathrm{~m}$ telescope at the European Southern Observatory in the Bessel $V$ and Gunn $r$ filters. Only 37 spots in 11 Schmidt fields have been observed during this run due to partial photometric weather conditions. They complete those observed a year before within the same context using the Toronto telescope at the Las Campanas Observatory (Infante et al. 1996).

\section{CCD Observations}

Both the large number of planned CCD exposures and the need to observe medium to faint stars (which are unsaturated on the ESO survey Schmidt emulsions) led us to make use of a medium aperture telescope, so that our observational program can be achieved within a few photometric nights. One run of observations was therefore conducted during 3 nights in May 1994 at ESO, La Silla, using the $2.2 \mathrm{~m} \mathrm{ESO/MPI}$ telescope with the EFOSC 2 system. The $1061 \times 1040$ CCD chip of the imaging camera (ESO CCD \#19) provides us with $1024 \times 1024$ images corresponding to a field of view of $5.6^{\prime} \times 5.6^{\prime}$ with a $\sim 0.326^{\prime \prime}$ resolution (pixel size: $19 \mu \mathrm{m}$ ). Bessel $V$ and Gunn $r$ filters were available on the filter wheel.

Several Landolt (1992) sequences of standard stars with different colours were observed three times each night at various air masses for calibration purposes. Namely, fields SA104, PG1323, PG1525, PG1657, SA110 and Mark A were used to determine the coefficients connecting the instrumental CCD magnitudes to the standard $V, R$ photometric system. The magnitude range of the measured stars is $11<R<16$ with a colour range of $-0.1<(V-R)<1.4$. The integration time for these bright objects was set to $3 \mathrm{~s}$ for focussed exposures.

The CCD fields needed for the overall calibration project have been spaced to insure a quite accurate mapping of the photometric variations across each photographic Schmidt plate: centre of the plate, centres of diagonals, and 3 spots along each edge. Five of these 13 spots per plate have been ranked the highest observational priority, so that an overview of the final result can be obtained with few exposures. In order to avoid saturations effects, the exact coordinates of the frames have been chosen with respect to regions devoid of stars brighter than $R=15$ using our photometric catalogue of the Shapley supercluster area. The large size of the current field of view ensures that a lot of stars are exposed each time, thereby securing the photometric zero point at these locations. The expo- sure time was set to $60 \mathrm{~s}$ for both $V$ and $r$ filters. Only the first night of this run and part of the third one had photometric conditions with sub-arcsec seeing, which led to 37 CCD observed fields (70 exposures over 208) in 11 Schmidt regions. Most of the frames which are pending to complete our calibration program have been recorded during further observational sessions held in 1995 and 1996 at the Las Campanas Observatory. These later data will be described in a forthcoming paper.

Table 1. Equatorial coordinates of the centres of the program fields

\begin{tabular}{|c|c|c|c|}
\hline $\begin{array}{l}\text { ESO/SERC } \\
\text { field }\end{array}$ & $\begin{array}{c}\text { Spot } \\
\#\end{array}$ & $\begin{array}{c}\alpha \\
(2000.0)\end{array}$ & $\begin{array}{c}\delta \\
(2000.0)\end{array}$ \\
\hline 324 & $\mathrm{e}$ & 132348.6 & -390236 \\
\hline 324 & $\mathrm{~g}$ & 133000.0 & -400303 \\
\hline 324 & $\mathrm{~m}$ & 131736.2 & $-42 \quad 1539$ \\
\hline 325 & $\mathrm{e}$ & 134905.5 & -390402 \\
\hline 325 & $\mathrm{~g}$ & 135506.7 & -400439 \\
\hline 325 & $\mathrm{~m}$ & 134309.4 & -420550 \\
\hline 382 & $\mathrm{~g}$ & 131516.3 & -350100 \\
\hline 383 & g & 133819.3 & -350002 \\
\hline 384 & $\mathrm{a}$ & 141356.8 & -353243 \\
\hline 384 & $\mathrm{e}$ & 135806.9 & -334614 \\
\hline 384 & $\mathrm{~g}$ & 140259.9 & $\begin{array}{lll}-35 & 03 & 17\end{array}$ \\
\hline 384 & $\mathrm{~h}$ & 135351.9 & -350100 \\
\hline 384 & i & 140815.1 & -361459 \\
\hline 384 & $\mathrm{~m}$ & 135316.1 & $\begin{array}{lll}-37 & 10 & 27\end{array}$ \\
\hline 444 & g & 132911.9 & -302123 \\
\hline 445 & $\mathrm{e}$ & 134542.4 & -285428 \\
\hline 445 & $\mathrm{~g}$ & 135133.7 & $\begin{array}{llll}-29 & 56 & 13\end{array}$ \\
\hline 445 & $\mathrm{i}$ & 135619.2 & $\begin{array}{llll}-3107 & 48\end{array}$ \\
\hline 445 & $\mathrm{~m}$ & 134022.7 & -322138 \\
\hline 446 & $\mathrm{e}$ & 140930.0 & -285946 \\
\hline 446 & g & 141401.5 & -300035 \\
\hline 446 & $\mathrm{~h}$ & 140524.5 & -300744 \\
\hline 446 & i & 141948.5 & -310800 \\
\hline 446 & $\mathrm{k}$ & 142501.9 & $\begin{array}{lll}-32 & 21 & 12\end{array}$ \\
\hline 446 & $\mathrm{~m}$ & 140414.8 & $\begin{array}{lll}-32 & 1804\end{array}$ \\
\hline 508 & $\mathrm{e}$ & 130953.2 & -234908 \\
\hline 508 & $\mathrm{~g}$ & 131431.6 & -250610 \\
\hline 508 & $\mathrm{~m}$ & 130450.6 & $\begin{array}{llll}-27 & 16 & 40\end{array}$ \\
\hline 509 & $\mathrm{e}$ & 133150.4 & -240847 \\
\hline 509 & $\mathrm{~g}$ & 133704.8 & -251706 \\
\hline 509 & $\mathrm{~m}$ & 132609.8 & -273348 \\
\hline 510 & $\mathrm{a}$ & 140808.0 & -223552 \\
\hline 510 & $\mathrm{e}$ & 135326.4 & -235139 \\
\hline 510 & $\mathrm{~g}$ & 135725.0 & -245648 \\
\hline 510 & $\mathrm{i}$ & 140235.2 & $\begin{array}{lll}-26 & 13 & 09\end{array}$ \\
\hline 510 & $\mathrm{k}$ & 140842.1 & -272243 \\
\hline 510 & $\mathrm{~m}$ & 134732.1 & -270849 \\
\hline
\end{tabular}




\section{Image processing and photometry}

The whole set of CCD frames has been processed using IRAF tasks for usual image processing operations and our own dedicated software for the fitting photometric procedures. First, the bias correction has been applied using a median averaged bias frame obtained from a blockaveraging of the initial data along the read-out axis. No systematics have been found within each night when compared to $10 \mathrm{mn}$ dark current exposures, and accordingly no scaling with respect to the exposure time has been made. Then, for each colour, the flat field correction involved a median frame obtained from the combination of several sky flat exposures taken either at dusk or at dawn. No master flat has been computed and no cosmic search has been performed due to the short integration time. Individual flat and bias exposures were used to determine the actual mean values of the gain and read-out noise of the CCD camera.

The standard stars were measured using the PHOT task for circular aperture photometry in an interactive way since only few bright objects had to be processed. An accurate centering of each individual object was computed by means of the implemented centroid algorithm, while the local sky background was computed from the mode (after iterative $3 \sigma$ rejections) of the pixel distribution inside a 10 pixel annulus with an inner radius of 23 pixels. The signal to background noise ratio is indeed high enough to get a correct total flux from this otherwise noisy estimator of the sky value. Instrumental magnitudes were computed using 7 aperture radii increasing by a factor $\sqrt{2}$ from $2 \sqrt{2}$ to $16 \sqrt{2}$ pixels. The aperture leading to the magnitude of each object was then chosen by eye from the light growing curve, which insures a correct aperture size and minimizes the magnitude error due to a background poor estimation. The equations which transform these CCD magnitudes to the standard system have been restricted to a zero-point $a_{0}$, airmass $a_{1}$ and colour $a_{2}$ terms according to:

$$
\begin{aligned}
& V=V_{\mathrm{CCD}}+a_{0, V}+a_{1, V} X+a_{2, V}(V-R) \\
& R=r_{\mathrm{CCD}}+a_{0, r}+a_{1, r} X+a_{2, r}(V-R) .
\end{aligned}
$$

We obtained the set of coefficients $\left\{a_{i}\right\}$ from a leastmean-square fitting program where the very few spurious measurements have been hand-flagged and rejected. The residual scatters for the first night ( $\simeq 40$ standard stars) are $\sigma_{V}^{(1)} \sim 0.03^{\mathrm{m}}$ and $\sigma_{R}^{(1)} \sim 0.04^{\mathrm{m}}$ in the $V$ and $R$ bands respectively. Only 9 stars are available for the third night due to the restricted duration of photometric conditions. The residual scatters we got are $\sigma_{V}^{(3)} \sim 0.02^{\mathrm{m}}$ and $\sigma_{R}^{(3)} \sim$ $0.05^{\mathrm{m}}$.

Because of the large number of objects per frame, the set of program fields needs to be processed automatically. We obtained the positions of the objects on the $r$ frames by using the IRAF task DAOFIND, which looks for local maxima in the brightness enhancements resulting from a convolution of the original data with an elliptical Gaussian function. Critical parameters for a successful detection are the full width at half maximum, the ellipticity, the roundness, and the sharpness of the searched astronomical objects. These morphological characteristics have been set for stars by examining their shape mean values on each frame, as well as the detection threshold by computing the standard deviation of the sky background. Note, however, that counts complete down to the detection limit was not the goal to reach. We are only looking for not very faint secondary calibrators. The aperture photometry on these object lists was then performed using PHOT in batch mode. As two checks confirmed it, the same list was valid for the $V$ and $r$ exposures since they had always been taken one after another without stoping the telescope guiding. However, an interactive determination of the instrumental magnitude from the set of aperture estimates could not now be considered due to the large number of objects. Thus, we decided for each observing night to compute the empirical law giving the best magnitude estimate for a given luminosity. To do so we processed in an interactive manner one field, chose for each object what seemed to be the "best" magnitude among the seven available values just like for the standard stars, and performed a first-order polynomial error-weighted fit between the sets of aperture radii and magnitudes. Comparisons of the resulting magnitude determinations with the somewhat subjective visual ones showed a good agreement: most of the time, the difference in aperture radius does not exceed one aperture and the machine selected magnitude is within the quoted error of the human selected choice. Therefore the fits obtained in the $V$ and $r$ bands were applied to the output files generated by PHOT in order to get the total instrumental magnitudes for the program objects. Photometric errors were also computed automatically by $\mathrm{PHOT}$ from the $\mathrm{S} / \mathrm{N}$ ratios based on the gain and read-out-noise of the CCD and on the flux and local sky values. Finally, the equations linking instrumental CCD and standard photometric systems were used with the observational $(V-r)$ colour indices to obtain the lists of the final corrected total magnitudes. These later were plotted against the instrumental values as a last check to flag objects which do not follow the main tendancy and remove them after a visual inspection of the CCD frame (bad measure due to close bright neighbours, lack of the counterpart in the $V$ photometric band, peculiar objects, etc.).

\section{Results}

Table 1 lists the actual coordinates of the centre of each program field together with their identification with respect to the ESO/SERC Schmidt field number and their position on the plate. Stars have been mostly detected on each frame by the DAOFIND task due to the 
parameters setting and to the low galactic latitude, but obviously some galaxies are also present, especially at magnitude levels where discrimination between stars and galaxies is difficult. Each primary catalogue includes about 200 star candidates with $V$ and $R$ magnitudes and photometric errors down to the detection limit $R \simeq 23$. The detection limit on the $\operatorname{ESO}(\mathrm{R})$ survey is $\sim 21^{\mathrm{m}}$, which corresponds mostly to CCD objects with a magnitude error in $R$ smaller than $0.1^{\mathrm{m}}$ and with a large enough area to minimize contamination effects from galaxy misidentifications. For calibration of our ESO (R) scans, we decided to secure such an accuracy by limiting the photometric catalogues to this magnitude limit in $R$. These final CCD sequences are listed in Tables 2 to 38 . Individual coordinates are computed from the position offsets to the centre of the observed field checked against objects measured on the scans. Their accuracy is better than $5^{\prime \prime}$.

Acknowledgements. Financial support from the FranceChile cooperation program C93U02 managed by the ECOS and CONICYT committes is gratefully acknowledged. L. Infante acknowledges financial support from FONDECYT grant 1930570. H. Quintana gratefully acknowledges support from FONDECYT grant 1930572 and the award of a Presidential Chair in Science. E. Slezak is grateful to G. Mars for his technical assistance concerning the data reductions.

\section{References}

Bardelli S., Zucca E., Vettolani G., Zamorani G., Scaramella R., et al., 1994, MNRAS 267, 665

Bunclark P.S., Irwin M.J., 1983, in Symp. on Statistical Methods in Astronomy, ESA SP-201, p. 195

Cunow B., 1993, A\&AS 97, 541

Infante L., Slezak E., Quintana H., 1996, A\&AS (submitted)

Landolt A.U., 1992, AJ 104, 340

Quintana H., Ramirez A., Melnick J., Raychaudhury S., Slezak E., 1995, AJ 110, 463

Raychaudhury S., 1989, Nat 342, 251 